\title{
Measuring the cluster magnetic field power spectra from Faraday rotation maps of Abell 400, Abell 2634 and Hydra A
}

\author{
C. Vogt and T. A. Enßlin
}

\author{
Max-Planck-Institut für Astrophysik, Karl-Schwarzschild-Str. 1, Postfach 1317, 85741 Garching, Germany \\ Received 10 June 2003 / Accepted 4 September 2003
}

\begin{abstract}
We apply a novel technique of Faraday Rotation measure (RM) map analysis to three galaxy clusters, Abell 400, Abell 2634 and Hydra A, in order to estimate cluster magnetic field strengths, length scales and power spectra. This analysis essentially a correlation analysis - is based on the assumption that the magnetic fields are statistically isotropically distributed across the Faraday screen. We investigate the difficulties involved in the application of the analysis to observational data. We derive magnetic power spectra for three clusters, i.e. Abell 400, Abell 2634 and Hydra A, and discuss influences on their shapes caused by the observational nature of the data such as limited source size and resolution. We successfully apply various tests to validate our assumptions. We show that magnetic fluctuations are probed on length scales ranging over at least one order of magnitude. Using this range for the determination of magnetic field strength of the central cluster gas yields $3 \mu \mathrm{G}$ in Abell 2634, $6 \mu \mathrm{G}$ in Abell 400 and $12 \mu \mathrm{G}$ in Hydra $\mathrm{A}$ as conservative estimates. The magnetic field autocorrelation length $\lambda_{B}$ was determined to be $4.9 \mathrm{kpc}$ for Abell 2634, $3.6 \mathrm{kpc}$ for Abell 400 and $0.9 \mathrm{kpc}$ for Hydra A. We show that the $R M$ autocorrelation length $\lambda_{R M}$ is larger than the magnetic field autocorrelation length $\lambda_{B}$ - for the three clusters studied, we found $\lambda_{R M} \simeq 2 \ldots 4 \lambda_{B}-$ and thus, they are not equal as often assumed in the literature. Furthermore, we investigate in a response analysis if it is possible to determine spectral slopes of the power spectra. We find that integrated numbers can be reliably determined from this analysis but differential parameters such as spectral slopes have to be treated differently. However, our response analysis results in spectral slopes of the power spectra of spectral indices $\alpha=1.6$ to 2.0 suggesting that Kolmogorov spectra $(\alpha=5 / 3)$ are possible but flatter spectral slopes than $\alpha=1.3$ can be excluded.
\end{abstract}

Key words. radiation mechanism: non-thermal - galaxies: active - interplanetary medium - galaxies: cluster: general radio continuum: general

\section{Introduction}

Clusters of galaxies are inhabited by magnetised plasma. The detection of diffuse synchrotron emission observed as cluster radio halos and radio relics is but one piece of evidence for the existence of cluster wide magnetic fields (for a review, see Carilli \& Taylor 2002). Still, their strengths, their structures and their origin remains to be unveiled.

Although the cluster wide magnetic fields are not believed to influence the dynamics directly in most clusters because the magnetic pressure is estimated to be too small compared to the thermal pressure, they are still important for the understanding of the physics of the intra-cluster gas. Indirectly they are likely an important cluster gas ingredient, e.g. cluster magnetic fields suppress heat conduction as observed in cluster cold fronts (e.g. Markevitch et al. 2000; Vikhlinin et al. 2001).

There are different methods to gain information about magnetic fields. One is to use the synchrotron radiation observed as radio halos or relics to estimate the field strength. Energy equipartition arguments for these clusters lead to strengths of

Send offprint requests to: C. Vogt,

e-mail: cvogt@mpa-garching.mpg.de about $\sim 0.1 \ldots 2 \mu \mathrm{G}$ (e.g. Feretti 1999; Brunetti et al. 2001). Another possibility is the comparison of their synchrotron radiation fluxes with inverse Compton X-ray emission leading to lower limits to the field strengths of about $0.2 \ldots 1 \mu \mathrm{G}$ (e.g. Harris \& Grindlay 1979; Rephaeli et al. 1987; Enßlin \& Biermann 1998; Fusco-Femiano et al. 1998; Bagchi et al. 1998).

A different method to gain knowledge about magnetic fields is the observation of the Faraday rotation effect which arises whenever polarised radio emission passes through a magnetised medium, causing its plane of polarisation to rotate if the magnetic field component parallel to the direction of the light propagation is nonzero. If the Faraday active medium is external to the source of the polarised radio emission one expects the rotation to be proportional to the squared wavelength. The proportionality constant is called the Faraday rotation measure $(R M)$ which can be evaluated in terms of the line of sight integral over the product of the electron density and the magnetic field component along the line of sight.

Recently, Rudnick \& Blundell (2003) claimed to have evidence for the $R M$ producing fields being local to the polarised radio source as suggested by Bicknell et al. (1990). However, 
this conclusion was based on a biased statistic without performing a proper null-experiment (Enßlin et al. 2003). Furthermore, the assumption that the Faraday rotation is produced in the intra-cluster gas is also supported by independent evidence. We mentioned already some of this evidence, the cluster wide diffuse radio emission observed as radio halos and relics. Further evidence is the observation of the Laing-Garrington effect. It manifests itself by an asymmetric Faraday depolarisation of double radio lobes located within galaxy clusters (Garrington et al. 1988; Laing 1988). This asymmetry is caused by different path lengths for radio emission through the Faraday active medium between source and observer as suggested by Garrington \& Conway (1991). This effect could also be explained by an asymmetric mixing layer being thin close to the head side of a FR II while becoming thicker on the back-flow side of it. However, this explanation fails to give a convincing explanation for the large $R M$ and depolarisation asymmetry observed for the FR I source Hydra A (Taylor \& Perley 1993).

Further independent evidence for cluster wide magnetic fields is provided by a recent statistical $R M$ investigation of point sources performed by Clarke et al. (2001). It revealed larger $R M$ values for sources observed through the intra-cluster gas in comparison to a control sample of sources where no intra-cluster gas was located between source and observer. This suggests that the enhancement of the $R M$ towards the cluster centre results most probably from the magnetised cluster gas.

Thus, we believe that the analysis of Faraday rotation maps of extended polarised radio sources located behind or embedded in galaxy clusters allows us to understand the strength and to get some hints about the structure of cluster magnetic fields.

A naive analysis of such maps is to estimate a minimum field strength by assuming a constant magnetic field throughout the cluster along the line of sight. However, close inspection of $R M$ maps of extended extragalactic radio sources reveals a patchy structure on small scales $(5-20 \mathrm{kpc})$ which indicates that the fields are tangled. An improved analysis of the $R M$ maps assumes the Faraday screen consists of cells which have a constant size and a constant magnetic field strength but the field direction varies randomly from cell to cell. The mean $R M$ which would be produced by such a screen builds up in a random walk and thus has a zero mean but a non vanishing dispersion. This dispersion will be proportional to the square root of the number of cells along the line of sight, the cell size, the electron density profile and the magnetic field strength as shown by Felten (1996) and Feretti et al. (1995). Using these proportionalities magnetic field strengths of the order of a few $\mu \mathrm{G}$ for non-cooling flow clusters, e.g. Coma (Feretti et al. 1995), A 119 (Feretti et al. 1999), 3C 129 (Taylor et al. 2001), A 514 (Govoni et al. 2001), A 400 \& A 2634 (Eilek \& Owen 2002), and of the order of a few $10 \mu \mathrm{G}$ for cooling flow clusters, e.g. Cygnus A (Dreher et al. 1987), 3C 295 (Perley \& Taylor 1991), A 1795 (Ge \& Owen 1993), Hydra A (Taylor \& Perley 1993), were derived.

However, there are some drawbacks of the cell model. First of all, the divergence of the magnetic field in such a Faraday screen would be nonzero which contradicts Maxwell's equations. Secondly, a spectrum of scales for the cell sizes is more likely than a single scale.
Another important issue in previous analyses is the assumption that the $R M$ ordering scale read off $R M$ maps is equivalent to the magnetic field's characteristic length scale, the autocorrelation length $\lambda_{B}$. This might have lead to underestimations of field strengths in the past since the equation often used for the derivation of the magnetic field strength is

$$
\left\langle B_{z}^{2}\right\rangle=\frac{\left\langle R M^{2}\right\rangle}{a_{0}^{2} n_{\mathrm{e}}^{2} L \lambda_{z}}
$$

where $\left\langle R M^{2}\right\rangle$ is the $R M$ dispersion, $L$ is the depth of the Faraday screen, $a_{0}=e^{3} /\left(2 \pi m_{\mathrm{e}}^{2} c^{4}\right), n_{\mathrm{e}}$ is the electron density and $\lambda_{z}$ is a characteristic length scale of the fields. At this point it becomes clear that the definition of the characteristic length scale $\lambda_{z}$ is crucial for the derivation of magnetic field strength. The assumption of a constant magnetic field throughout the cluster leads to a definition of $\lambda_{z}=L$ and thus, $\left\langle B_{z}^{2}\right\rangle \propto L^{-2}$. The characteristic length scale for the cell model would be the size of each cell $\lambda_{z}=l_{\text {cell }}$. Another definition one could think of is the $R M$ ordering scale, $\lambda_{z}=\lambda_{R M}$. However, the correct length scale is the autocorrelation length $\lambda_{z}$ of the $z$-component of the magnetic field measured along the line of sight.

A purely statistical approach which incorporates the vanishing divergence of the magnetic field was recently developed by Enßlin \& Vogt (2003). This approach relies on the assumption that the fields are statistically isotropically distributed in Faraday screens such that the $z$-component is representative for all components. Starting from this assumption, a relation between the observationally accessible $R M$ autocorrelation function and the magnetic autocorrelation tensor is established in real and in Fourier space such that one gains access to the power spectrum of the magnetic field inhabited by the Faraday screen.

Enßlin \& Vogt (2003) demonstrated that in the case of isotropy the magnetic autocorrelation length $\lambda_{z}$ in Eq. (1) is given by $\lambda_{z}=3 / 2 \lambda_{B}$, where $\lambda_{B}$ is the 3 -dimensional magnetic autocorrelation length. Therefore, Eq. (1) becomes

$$
\left\langle B^{2}\right\rangle=3\left\langle B_{z}^{2}\right\rangle=\frac{2\left\langle R M^{2}\right\rangle}{a_{0}^{2} n_{\mathrm{e}}^{2} L \lambda_{B}} .
$$

The length scale $\lambda_{B}$ can be estimated from the measured $R M$ power spectrum.

Here we apply this statistical analysis to observational data by reanalysing the Faraday rotation measure maps of three extended extragalactic radio sources: Hydra A (Taylor \& Perley 1993), 3C 75 and 3C 465 (Eilek \& Owen 2002) which were kindly provided by Greg Taylor, Frazer Owen, and Jean Eilek, respectively.

In Sect. 2, we outline shortly the idea of our approach, the real and the Fourier space analysis and what possibilities are given to test if the assumptions made are reasonable. Furthermore, we state the equations which were used in the analysis while their derivation and more detailed discussion can be found in Enßlin \& Vogt (2003). In Sect. 3, the application of these approaches to the observational data and the difficulties involved are described. Emphasis is given to a critical discussion of the strengths and limits of the approach. The results are 
presented and discussed in Sect. 4. Finally, the conclusions are drawn in the last Sect. 5.

Throughout the rest of the paper we assume a Hubble constant of $H_{0}=70 \mathrm{~km} \mathrm{~s}^{-1} \mathrm{Mpc}^{-1}, \Omega_{m}=0.3$ and $\Omega_{\Lambda}=0.7$ in a flat universe. All equations follow the notation of Enßlin \& Vogt (2003).

\section{Method}

\subsection{Idea}

We assume that the magnetic fields in galaxy clusters are isotropically distributed throughout the Faraday screen. If one samples these field distributions over a sufficiently large volume they can be treated as statistically homogeneous and statistically isotropic. This means that the statistical average over any field quantity will not be influenced by the geometry or the exact location of the volume sampled.

One of the magnetic field quantities which is of interest here is its autocorrelation function since this function can be used to calculate its correlation length and field strength. Furthermore, the autocorrelation function is equivalent to the Fourier transform of the power spectrum as stated by the Wiener-Khinchin Theorem (WKT).

Since the magnetic field is a vector quantity, an autocorrelation tensor has to be considered rather than a function. The diagonal elements of this tensor are all equal in the case of magnetic isotropy which we assume as stated above. On the other hand, its off-diagonal terms consist of two numbers describing the symmetric and non-symmetric contribution. The divergence-freeness of magnetic fields $(\boldsymbol{\nabla} \cdot \boldsymbol{B}=0)$ couples the symmetric components of the tensor which means that the knowledge of a diagonal element ensures complete knowledge of the symmetric part of the tensor. Introducing now the scalar magnetic autocorrelation function $w(r)$ as the trace of the autocorrelation tensor $w(r)=\langle\boldsymbol{B}(\boldsymbol{x}) \cdot \boldsymbol{B}(\boldsymbol{x}+\boldsymbol{r})\rangle_{x}$, field quantities can be derived such as the magnetic autocorrelation length and average magnetic field energies using this function.

The non-symmetric part of the tensor represents the magnetic helicity. These off-diagonal terms of the tensor do not enter in the definition of the scalar magnetic autocorrelation function $w(r)$ and thus, do not have any effect on the quantities of interest listed above.

The observable considered in our approach is the Faraday rotation which is related to the projected magnetic field distribution along the line of sight. It is possible to establish a relation between the autocorrelation function of the $R M$ map and the autocorrelation function of the magnetic field as discussed in more detail in the next sections.

As stated above, the knowledge of the autocorrelation function gives access to the power spectra using the WKT. However, any phase information is lost by measuring power spectra. Therefore, it is possible that different realisations of magnetic field structure have the same power spectrum. This implies that not all information which might be contained in the $R M$ maps are accessible by the analysis presented. Correlation functions of higher order statistic are needed to access these information.
In the discussion so far, we ignored the fact that we are using observational data and that the $R M$ is also dependent on electron density and magnetic energy density on global scales which makes the introduction of a window function necessary. This window function can be interpreted as the sampling volume. It accounts for the limited size of the radio source and the global properties of the cluster gas such as the electron density and the average magnetic energy density profiles. A virtually statistical homogeneous magnetic field can be thought to be observed through this function.

The window function will be zero for points outside the volume which is situated in front of the radio source. It will scale with the electron density and the global magnetic field energy distribution within the Faraday screen between radio source and observer. Furthermore if wanted the window function can contain noise reducing data weighting schemes.

The overall effect of any finite window in real space is to smear out power in Fourier space. Furthermore, any analysis employing a window function will be sensitive to magnetic energies on scales smaller than the window size and insensitive to scales larger than the window size. Therefore, understanding the influence of the window function is crucial for the evaluation of the results obtained by applying of our analysis to observational data. Possibilities to assess its influence are given in Sect. 2.4.

\subsection{Real space analysis}

For a line of sight parallel to the $z$-axis and displaced by $\boldsymbol{x}_{\perp}$ from it, the Faraday rotation measure arising from polarised emission passing from the source at $z_{\mathrm{s}}\left(\boldsymbol{x}_{\perp}\right)$ through a magnetised medium to the observer located at infinity is expressed by

$R M\left(\boldsymbol{x}_{\perp}\right)=a_{0} \int_{z_{\mathrm{s}}\left(\boldsymbol{x}_{\perp}\right)}^{\infty} \mathrm{d} z n_{\mathrm{e}}(\boldsymbol{x}) B_{z}(\boldsymbol{x})$,

where $a_{0}=e^{3} /\left(2 \pi m_{\mathrm{e}}^{2} c^{4}\right), \boldsymbol{x}=\left(\boldsymbol{x}_{\perp}, z\right), n_{\mathrm{e}}(\boldsymbol{x})$ is the electron density and $B_{z}(\boldsymbol{x})$ is the magnetic field component parallel to the line of sight.

The autocorrelation function of the Faraday rotation map can be defined as

$C_{R M}\left(\boldsymbol{r}_{\perp}\right)=\left\langle R M\left(\boldsymbol{x}_{\perp}\right) R M\left(\boldsymbol{x}_{\perp}+\boldsymbol{r}_{\perp}\right)\right\rangle_{\boldsymbol{x}_{\perp}}$,

where the brackets mean the map average with respect to $\boldsymbol{x}_{\perp}$. However, an observed $R M$ map is limited in size which leads to noise in the calculation of the correlation function on large scales since less pixel pairs contribute to the correlation function for larger pixel separations. For suppressing this undersampling, the observable correlation function was chosen to be

$C_{R M}^{\mathrm{obs}}\left(r_{\perp}\right)=\frac{1}{A_{\Omega}} \int \mathrm{d}^{2} x_{\perp} R M\left(\boldsymbol{x}_{\perp}\right) R M\left(\boldsymbol{x}_{\perp}+\boldsymbol{r}_{\perp}\right)$,

where the area $A_{\Omega}$ of the region $\Omega$ for which $R M$ 's are actually measured is used as normalisation assuming that $R M\left(x_{\perp}\right)=0$ for $x_{\perp} \notin \Omega$. Furthermore, it is required that the mean $R M$ is zero since statistically isotropic divergence free fields have a mean $R M$ of zero to high accuracy. The non vanishing mean $R M$ in the observational data stems from foregrounds 
(e.g. the galaxy) in which we are not interested or has its origin in large scale fields to which the approach is insensitive by construction in order to suppress statistically under-sampled length scales.

As stated in Sect. 2.1, the virtually homogeneous magnetic field component can be thought to be observed through a window function $f(\boldsymbol{x})$ which describes the sampling volume. One would chose a typical position in the cluster $\boldsymbol{x}_{\text {ref }}$ (e.g. its centre) and define $n_{\mathrm{e} 0}=n_{\mathrm{e}}\left(\boldsymbol{x}_{\text {ref }}\right)$ and $\boldsymbol{B}_{0}=\left\langle\boldsymbol{B}^{2}\left(\boldsymbol{x}_{\text {ref }}\right)\right\rangle^{1 / 2}$. The window function can then be expressed as

$f(\boldsymbol{x})=\mathbf{1}_{\left\{\boldsymbol{x}_{\perp} \in \Omega\right\}} \mathbf{1}_{\left\{z \geq z_{\mathrm{s}}\left(\boldsymbol{x}_{\perp}\right)\right\}} h\left(\boldsymbol{x}_{\perp}\right) g(\boldsymbol{x}) n_{\mathrm{e}}(\boldsymbol{x}) / n_{\mathrm{e} 0}$,

where $\mathbf{1}_{\text {\{condition\} }}$ is equal to one if the condition is true and zero if not. The dimensionless average magnetic field profile $g(\boldsymbol{x})=$ $\left\langle\boldsymbol{B}^{2}(\boldsymbol{x})\right\rangle^{1 / 2} / B_{0}$ is assumed to scale with the density profile such that $g(\boldsymbol{x})=\left(n_{\mathrm{e}}(\boldsymbol{x}) / n_{\mathrm{e} 0}\right)^{\alpha_{B}}$. Reasonable values for the exponent $\alpha_{B}$ range between 0.5 and 1 as suggested by Dolag et al. (2001). The function $h(\boldsymbol{x})$ allows to introduce a suitable data weighting scheme and can be chosen to be unity if no data weighting is required.

As mentioned above, the magnetic autocorrelation function $w(r)$ can be defined as the trace of the magnetic field autocorrelation tensor $M_{i j}=\left\langle B_{i}(\boldsymbol{x}) B_{j}(\boldsymbol{x}+\boldsymbol{r})\right\rangle$ assuming isotropy $w(\boldsymbol{r})=\langle\boldsymbol{B}(\boldsymbol{x}) \cdot \boldsymbol{B}(\boldsymbol{x}+\boldsymbol{r})\rangle=\sum_{i} M_{i i}$, (e.g. Subramanian 1999). However, the Faraday rotation is also related to the magnetic field via Eq. (3). Therefore any $R M$ correlation contains also information on the autocorrelation of the magnetic field component along the line of sight

$$
\begin{aligned}
C_{\perp}\left(r_{\perp}\right) & =\int_{-\infty}^{\infty} \mathrm{d} r_{z} M_{z z}(\boldsymbol{r}) \\
& =\frac{1}{2} \int_{-\infty}^{\infty} \mathrm{d} r_{z} w\left(\sqrt{r_{\perp}^{2}+r_{z}^{2}}\right),
\end{aligned}
$$

where $\boldsymbol{r}=\left(\boldsymbol{r}_{\perp}, r_{z}\right)$. Taking the effect of the window function into account, we introduced $C_{\perp}\left(r_{\perp}\right)=\left\langle C_{R M}^{\mathrm{obs}}\left(\boldsymbol{r}_{\perp}\right)\right\rangle / a_{1}$, where $a_{1}=a_{0}^{2} n_{\mathrm{e} 0}^{2} L$. The parameter $L$ can be interpreted as the characteristic depth of the Faraday screen and can be expressed as $L=V_{[f]} / A_{\Omega}$, where $V_{[f]}$ is the probed effective volume $\int \mathrm{d}^{3} x f^{2}(\boldsymbol{x})$.

Using Eq. (8), one is now able to establish a relationship between the $R M$ autocorrelation function $C_{\perp}\left(r_{\perp}\right)$ and the magnetic autocorrelation function $w(r)$. This relationship can be expressed as

$$
\begin{aligned}
w(r) & =-\frac{2}{\pi r} \frac{\mathrm{d}}{\mathrm{d} r} \int_{r}^{\infty} \mathrm{d} y \frac{y C_{\perp}(y)}{\sqrt{y^{2}-r^{2}}} \\
& =-\frac{2}{\pi} \int_{r}^{\infty} \mathrm{d} y \frac{C^{\prime}(y)}{\sqrt{y^{2}-r^{2}}},
\end{aligned}
$$

where for the deprojection of Eq. (8) an Abel integral equation was used assuming $w(r)$ stays bounded for $r \rightarrow \infty$.

These two equations enable us to calculate not only the magnetic autocorrelation function but also to obtain directly the average magnetic energy density $\left\langle\varepsilon_{B}\right\rangle$ by using the relation $\left\langle\varepsilon_{B}\right\rangle=w(0) / 8 \pi$ and thus, we are able to determine the magnetic field strength by calculating the value of the magnetic autocorrelation function at point $r=0: w(r=0)=\left\langle B^{2}\right\rangle$.
The $R M$ autocorrelation length $\lambda_{R M}$ and the magnetic autocorrelation length $\lambda_{B}$ can be calculated by integrating the correlation functions:

$$
\begin{aligned}
\lambda_{R M} & =\int_{-\infty}^{\infty} \mathrm{d} r \frac{\left\langle R M\left(\boldsymbol{x}+r \boldsymbol{e}_{\perp}\right) R M(\boldsymbol{x})\right\rangle_{\boldsymbol{x}, \boldsymbol{e}_{\perp}}}{\left\langle R M^{2}(\boldsymbol{x})\right\rangle} \\
& =\int_{-\infty}^{\infty} \mathrm{d} r_{\perp} \frac{C_{\perp}\left(r_{\perp}\right)}{C_{\perp}(0)}=\pi \frac{\int_{-\infty}^{\infty} \mathrm{d} r r w(r)}{\int_{-\infty}^{\infty} \mathrm{d} r w(r)} \\
\lambda_{B} & =\int_{-\infty}^{\infty} \mathrm{d} r \frac{\langle B(\boldsymbol{x}+r \boldsymbol{e}) \cdot B(\boldsymbol{x})\rangle_{\boldsymbol{x}, \boldsymbol{e}}}{\left\langle B^{2}(\boldsymbol{x})\right\rangle} \\
& =\int_{-\infty}^{\infty} \mathrm{d} r \frac{w(r)}{w(0)}=2 \frac{C_{\perp}(0)}{w(0)}
\end{aligned}
$$

where $\boldsymbol{e}$ and $\boldsymbol{e}_{\perp}$ are 3- and 2-dimensional unit vectors, respectively, over which we are averaging.

\subsection{Fourier space analysis}

In Fourier space a similar formalism can be developed assuming statistical isotropy and relying on the $\boldsymbol{\nabla} \cdot \boldsymbol{B}=0$ condition. After performing a 2-dim Fourier transform ${ }^{1}$ of the $R M$ map, one can calculate the $R M$ autocorrelation in Fourier space:

$\hat{C}_{\perp}\left(k_{\perp}\right)=\frac{\left\langle\left|R \hat{M}\left(k_{\perp}\right)\right|^{2}\right\rangle}{a_{1} A_{\Omega}}$,

implied by the WKT which connects the 2-dimensional Fourier transformed $R M$ maps with the $R M$ autocorrelation function.

In Fourier space Eq. (8), the relationship between the $R M$ and the magnetic field autocorrelation function, can be expressed by

$\hat{C}_{\perp}\left(\boldsymbol{k}_{\perp}\right)=\hat{M}_{z z}\left(\boldsymbol{k}_{\perp}, 0\right)=\frac{1}{2} \hat{w}\left(\boldsymbol{k}_{\perp}, 0\right)$.

This equation states that the $k_{z}=0$ plane of the $M_{z z}$ component (see Eq. (7)) of the Fourier transformed magnetic autocorrelation tensor is accessible by the 2-dimensional Fourier transformation of the $R M$ map. Since all diagonal elements of the autocorrelation tensor are equal in the case of statistical isotropy, no more information is required in order to reconstruct the full magnetic autocorrelation.

The 3-dimensional power spectrum $\hat{w}(k) \mathrm{d}^{3} k$ is related to the 1-dimensional magnetic energy spectrum in the Fourier space $\varepsilon_{B}^{\mathrm{obs}}(k) \mathrm{d} k$ by

$\varepsilon_{B}^{\mathrm{obs}}(k)=\frac{k^{2} \hat{w}(k)}{2(2 \pi)^{3}}$,

where $\hat{w}(\boldsymbol{k})=\hat{w}(k)$ because of isotropy.

The combination of Eq. (15) and Eq. (17) leads to an equation which relates the magnetic energy spectrum directly to the Fourier transformed $R M$ map

$\varepsilon_{B}^{\mathrm{obs}}(k)=\frac{k^{2}}{a_{1} A_{\Omega}(2 \pi)^{4}} \int_{0}^{2 \pi} \mathrm{d} \phi\left|R \hat{M}\left(k_{\perp}\right)\right|^{2}$,

1 Defining the Fourier transformation for a $n$-dimensional function $F(\boldsymbol{x})$ such that:

$\hat{F}(k)=\int \mathrm{d}^{n} x F(\boldsymbol{x}) \mathrm{e}^{\mathrm{i} \boldsymbol{k} \cdot \boldsymbol{x}} ; F(\boldsymbol{x})=\frac{1}{(2 \pi)^{n}} \int \mathrm{d}^{n} k \hat{F}(\boldsymbol{k}) \mathrm{e}^{-\mathrm{i} \boldsymbol{k} \cdot \boldsymbol{x}}$. 
which is equivalent to averaging over rings in $k$-space. Therefore integration over $k$ yields the average magnetic energy density $\varepsilon_{B}^{\mathrm{obs}}=\int_{0}^{\infty} \mathrm{d} k \varepsilon_{B}^{\mathrm{obs}}(k)$ and thus, leads to a magnetic field strength estimate.

Other quantities like the correlation length scales, $\lambda_{B}$ and $\lambda_{R M}$, can also be calculated via integrating over the correlation function:

$$
\begin{aligned}
\lambda_{R M} & =2 \frac{\int_{0}^{\infty} \mathrm{d} k \hat{w}(k)}{\int_{0}^{\infty} \mathrm{d} k k \hat{w}(k)} \\
\lambda_{B} & =\pi \frac{\int_{0}^{\infty} \mathrm{d} k k \hat{w}(k)}{\int_{0}^{\infty} \mathrm{d} k k^{2} \hat{w}(k)} .
\end{aligned}
$$

From these two equations, it is obvious that the $R M$ autocorrelation length $\lambda_{R M}$ has a much larger weight on large-scale fluctuations than the magnetic correlation length $\lambda_{B}$. Therefore one expects that $\lambda_{R M}>\lambda_{B}$ in the natural case of a broadly peaked magnetic power spectrum.

The autocorrelation function and the power spectrum are related by a Fourier transform as stated by the WKT, therefore one can derive a relation for the magnetic autocorrelation function $w(r)$ in terms of the power spectrum $\hat{w}(k)$

$w(r)=\frac{4 \pi}{(2 \pi)^{3}} \int_{0}^{\infty} \mathrm{d} k k^{2} \hat{w}(k) \frac{\sin k r}{k r}$.

The derivation of the function $w(r)$ employing the power spectra $\hat{w}(k)$ can be used as a good test for proper numerics, since it can be compared to the deprojected magnetic autocorrelation function $w(r)$ determined in real space.

\subsection{Testing the model and assessing the influence of the window function}

There are different methods to assess the results obtained using the approaches in real and Fourier space described above. Besides the comparison of the numbers determined for the characteristic length scales and magnetic field strengths in both spaces, there is also the possibility to evaluate the quality of the models used for the magnetic energy density and the location of the radio emitting source within or behind the Faraday screen. For this purpose a $\chi^{2}$-test function can be introduced such that

$\chi^{2}\left(\boldsymbol{x}_{\perp}\right)=\frac{R M\left(\boldsymbol{x}_{\perp}\right)^{2}}{\left\langle R M\left(\boldsymbol{x}_{\perp}\right)^{2}\right\rangle}$,

where the expected $R M$ dispersion is defined as $\left\langle R M\left(\boldsymbol{x}_{\perp}\right)^{2}\right\rangle=$ $0.5 a_{0}^{2} n_{\mathrm{e} 0}^{2} B_{0}^{2} \lambda_{B} \int_{-\infty}^{\infty} \mathrm{d} z f^{2}(\boldsymbol{x})$. If one averages this function radially and examines it for any tendency in its spatial distribution, one has a first test of the model. If there are any large scale trends observed one should reconsider the model used. Furthermore, the integration of this function should result in a value close to unity

$\chi_{a v}^{2}=\frac{1}{A_{\Omega}} \int \mathrm{d} x_{\perp}^{2} \chi^{2}\left(\boldsymbol{x}_{\perp}\right) \approx 1$,

if the choice of the model is a suitable description of the global magnetic energy distribution and the geometry of the source.
Higher values of $\chi_{a v}^{2}$ would arise for unsuited models and one would have to refine the model parameters.

The isotropy which is an important assumption for our analysis can be assessed by the inspection of the Fourier transformed $R M$ maps. The assumption of isotropy is valid in the case of a spherically symmetric distribution of $|R \hat{M}(k)|^{2}$.

A completely different problem is the assessment of the influence of the window function on the shape of the magnetic power spectrum and thus, on the measured magnetic energy spectrum. As stated above, the introduction of a finite window function has the effect to smear out the power spectrum and Eq. (16) becomes

$\hat{C}_{\perp}^{\exp }\left(\boldsymbol{k}_{\perp}\right)=\frac{1}{2} \int \mathrm{d}^{3} q \hat{w}(q) \frac{q_{\perp}^{2}}{q^{2}} \frac{\left|\hat{f}\left(\boldsymbol{k}_{\perp}-\boldsymbol{q}\right)\right|^{2}}{(2 \pi)^{3} V_{[f]}}$.

The term $q_{\perp}^{2} / q^{2} \leq 1$ in the expression above states some loss of magnetic power. Without this term, the expression would describe the redistribution of magnetic power within Fourier space, where in this case the magnetic energy would be conserved.

However, the expression can be employed as an estimator of the response of the observation to the magnetic power on a given scale $p$ by setting $\hat{w}(q)=\delta(q-p)$ in the above equation. For an approximate treatment of a realistic window and a spherical data average, one can derive

$\hat{w}_{p}^{\exp }\left(k_{\perp}\right)=\frac{2 p}{\pi} \int_{\left|k_{\perp}-q\right|}^{k_{\perp}+q} \frac{q \int_{0}^{2 \pi} \mathrm{d} \phi W_{\perp}(q(\cos \phi, \sin \phi))}{\sqrt{4 q^{2} p^{2}-\left(q^{2}+p^{2}-k_{\perp}^{2}\right)^{2}}}$.

Here the projected Fourier window was introduced

$W_{\perp}\left(\boldsymbol{k}_{\perp}\right)=\frac{\left|\hat{f}_{\perp}\left(\boldsymbol{k}_{\perp}\right)\right|^{2}}{(2 \pi)^{2} A_{\left[f_{\perp}\right]}}$,

where $A_{\left[f_{\perp}\right]}=\int \mathrm{d}^{2} x_{\perp} f_{\perp}^{2}\left(\boldsymbol{x}_{\perp}\right)$ while defining the projected window function as $f_{\perp}\left(\boldsymbol{x}_{\perp}\right)=\int \mathrm{d} z f^{2}(\boldsymbol{x}) V_{[f]} / A_{\Omega}$.

One can also require for the input power spectrum a field strength by setting $\hat{w}(q)=2 \pi^{2}\left(B^{2} / p^{2}\right) \delta(q-p)$ in Eq. (24). The resulting power spectrum can be treated as an observed power spectrum and thus, a magnetic field strength $B_{\exp }$ can be derived by integration following Eq. (17). The comparison of $B$ and $B_{\exp }$ for the different $p$-scales yields a further estimate for reliable ranges in $\mathrm{k}$-space.

So far the response power spectrum was derived for an underlying magnetic spectrum located only on scales $p$. One could also add the particular response functions such that the actual observed power spectrum $\hat{w}^{\text {obs }}(k)$ would be matched. Typical power spectra, e.g. for turbulence, show a power law behaviour over several orders of magnitude. Therefore, one would choose to weight the different response functions $\hat{w}_{p}^{\exp }\left(k_{\perp}\right)$ derived using Eq. (25) by a power law while integrating over them

$\hat{w}^{\exp }(k)=w_{0} \int_{p_{\min }}^{p_{\max }} \mathrm{d} p \hat{w}_{p}^{\exp }\left(k_{\perp}\right)\left(\frac{p}{k_{0}}\right)^{-\alpha-2}$,

where $w_{0}$ represents a normalisation constant, $k_{0}$ a typical $k$ space length scale and $p_{\min }$ and $p_{\max }$ are upper and lower cutoffs for the integration over the response functions. Ideally, 
one would choose the normalisation constant $w_{0}=c_{0} B_{0}^{2} / k_{0}^{3}$, where $c_{0}$ is chosen such that $\int \mathrm{d} k^{3} \hat{w}^{\exp }(k)=B_{0}^{2}$. One would vary the spectral index $\alpha$ of the power law, $B_{0}$ or $w_{0}$ and the lower cutoff of the integration $p_{\text {min }}$ in order to match the two functions, $\hat{w}^{\mathrm{obs}}(k)$ and $\hat{w}^{\exp }(k)$.

One has direct access to the average magnetic energy density $\varepsilon_{B}$ by integrating Eq. (27) which results in the analytic expression

$\varepsilon_{B}=\frac{w_{0} k_{0}^{3}}{2(2 \pi)^{3}(\alpha-1)}\left[\left(\frac{p_{\min }}{k_{0}}\right)^{1-\alpha}-\left(\frac{p_{\max }}{k_{0}}\right)^{1-\alpha}\right]$.

Following the definition of the magnetic autocorrelation length $\lambda_{B}$ (Eq. (20)), one can derive an expression for this length using the parameters determined in the analysis above

$\lambda_{B}=\pi \frac{\alpha-1}{\alpha} \frac{p_{\min }^{-\alpha}-p_{\max }^{-\alpha}}{p_{\min }^{1-\alpha}-p_{\max }^{1-\alpha}}$

In the calculation of the expected response $\hat{C}_{\perp}^{\exp }\left(\boldsymbol{k}_{\perp}\right)$ and thus, $\hat{w}^{\exp }\left(\boldsymbol{k}_{\perp}\right)$ to an underlying magnetic power spectrum $\hat{w}(k)$ seen through some window $W(\boldsymbol{k})$ as expressed by Eq. (25) (or Eqs. (51), (67), (68) of Enßlin \& Vogt 2003), it has not been considered that the mean $R M$ is subtracted from the observed $R M$ maps before our analysis is applied to them in order to remove contributions from foreground $R M$ screens (e.g. the galaxy). We take this into account by noting that the $R M$ value of a pixel at $\boldsymbol{x}_{\perp}$ in the map will be changed only in the case of $\boldsymbol{x}_{\perp} \in \Omega$, which can be written as

$R M\left(x_{\perp}\right) \rightarrow R M^{*}\left(x_{\perp}\right)=R M\left(x_{\perp}\right)-\mathbf{1}_{\left\{x_{\perp} \in \Omega\right\}} \int \frac{\mathrm{d}^{2} x_{\perp}^{\prime}}{A_{\Omega}} R M\left(x_{\perp}^{\prime}\right)$.

Using the properties of the Fourier transform of a function with compact support, one can show that

$\hat{C}_{R M^{*}}^{\exp }\left(\boldsymbol{k}_{\perp}\right)=\hat{C}_{R M}^{\exp }\left(\boldsymbol{k}_{\perp}\right)-\hat{C}_{R M}^{\exp }\left(\mathbf{0}_{\perp}\right)\left|\Delta_{\Omega}\left(\boldsymbol{k}_{\perp}\right)\right|^{2}$,

where

$\Delta_{\Omega}\left(\boldsymbol{k}_{\perp}\right)=\frac{1}{A_{\Omega}} \int \mathrm{d}^{2} x_{\perp} \mathbf{1}_{\left\{x_{\perp} \in \Omega\right\}} \mathrm{e}^{\mathrm{i} \boldsymbol{k}_{\perp} \cdot \boldsymbol{x}_{\perp}}$,

and $\hat{C}_{R M}^{\mathrm{exp}}\left(\boldsymbol{k}_{\perp}\right)$ is the expected, uncorrected response to $\hat{w}(k)$. The linearity of the problem ensures that this correction is valid for any input power spectra $\hat{w}(k)$ (as long as $\hat{C}_{R M}^{\exp }\left(\boldsymbol{k}_{\perp}\right)$ is calculated from it) which could be a delta-function, a power law, or could have any other shape. Similarly, we can write for the spherically averaged expected observed power spectrum

$\hat{w}_{*}^{\exp }(k)=\hat{w}^{\exp }(k)-\hat{w}^{\exp }(0) \frac{1}{2 \pi} \int_{0}^{2 \pi} \mathrm{d} \phi \mid \Delta_{\Omega}\left(\left.k(\cos \phi, \sin \phi)\right|^{2}\right.$,

where $\hat{w}^{\exp }(k)$ is given by Eq. (25).

The subtraction of the mean $R M$ does not only remove unwanted homogeneous foregrounds, it also ensures that $\hat{w}_{*}^{\exp }(k)$ (or $\hat{C}_{R M^{*}}^{\exp }\left(\boldsymbol{k}_{\perp}\right)$ ) vanishes at the origin $\hat{w}_{*}^{\exp }(0)=0$ (similarly $\left.\hat{C}_{R M^{*}}^{\exp }\left(\mathbf{0}_{\perp}\right)=0\right)$. For a correct behaviour of the responses at low $k$ this correction is therefore crucial and will be taken into account in the following.

\section{Application to existing rotation measure maps of Galaxy clusters}

Before we applied our analysis to the data any mean $R M$ value was subtracted from the $R M$ maps. This is necessary as mentioned above, since we assume statistically isotropic fields and therefore we have to remove any $R M$ foreground which would affect our analysis. Furthermore, very noisy pixels and pixel areas exhibiting huge differences in the $R M$ values on scales smaller than the beamsize were removed from the data to decrease the possible influences of observational artifacts.

The origin of the $z$-axis was placed in the cluster centre and was used as reference point $\boldsymbol{x}_{\text {ref }}$ for the global distribution of electron density and magnetic energy. The latter was assumed to scale with the electron density such that $\left\langle\boldsymbol{B}^{2}(\boldsymbol{x})\right\rangle^{1 / 2} / B_{0}=$ $\left(n_{\mathrm{e}}(\boldsymbol{x}) / n_{\mathrm{e} 0}\right)^{\alpha_{B}}$. Unless stated otherwise, the parameter $\alpha_{B}$ was chosen to be unity. For the electron density distribution a standard $\beta$-profile ${ }^{2}$ was used.

\section{1. $3 C 75,3 C 465$ \& Hydra $A$ - the data}

The radio source 3C 465 (or NGC 7720) is located in the Abell cluster 2634 of richness class I. The redshift of the object is 0.0302 (Wegner et al. 1999). A detailed X-ray study of this cluster was performed by Schindler \& Prieto (1997) using ROSAT PSPC data. They derive for the density profile a core radius of 4.9 arcmin, a $\beta$ of 0.79 and an electron density at the cluster centre of $n_{\mathrm{e} 0}=0.0012 \mathrm{~cm}^{-3}$. Their analysis revealed indications of a weak cooling flow in the cluster centre. The scenario of a weak cooling flow in Abell 2634 is also supported by White (2000) who analysed ASCA X-ray data.

The radio galaxy $3 \mathrm{C} 75$ which is in the centre of Abell 400 also of richness class I has a redshift of 0.02315 (de Vaucouleurs et al. 1991). The X-ray properties of the cluster have been studied by Reiprich \& Böhringer (2002) using ROSAT PSPC data. They determine for the gas density profile a core radius of 3.9 arcmin, a $\beta$ of 0.54 and an electron density at the cluster centre of $n_{\mathrm{e} 0}=0.0016 \mathrm{~cm}^{-3}$. There are no indications of a cooling flow in Abell 400 (White 2000).

The details of the radio data reduction of the $R M$ maps for the two sources above can be found in Eilek \& Owen (2002). The typical $R M$ values observed for them ranging between $-250 \mathrm{rad} \mathrm{m}^{-2}$ and $250 \mathrm{rad} \mathrm{m}^{-2}$. The beamsize for the map of 3C 75 (Abell 400) is 3 arcsec which translates into $1.4 \mathrm{kpc}$ and for 3C 465 (Abell 2634) the beamsize is 3.8 arcsec which is equivalent to $2.3 \mathrm{kpc}$ for the cosmology chosen.

Furthermore, it was assumed that the source plane is parallel to the observer plane since no indication for the Laing-Garrington effect was found.

Hydra A (or 3C 218) is an extended extragalactic radio source located at a redshift of 0.0538 (de Vaucouleurs et al. 1991) in the cluster Abell 780 of richness class 0. However, we will refer to it hereafter as Hydra A cluster. Detailed Xray studies have been performed on it (e.g. Ikebe et al. 1997; Peres et al. 1998; David et al. 2001). For the derivation of the

${ }^{2}$ Defined as $n_{\mathrm{e}}(r)=n_{\mathrm{e} 0}\left(1+\left(r / r_{\mathrm{c}}\right)^{2}\right)^{-\frac{3}{2} \beta}$, where $r_{\mathrm{c}}$ is called the core radius. 
electron density profile parameter, we relied on the work by Mohr et al. (1999) done for ROSAT PSPC data while using the deprojection of X-ray surface brightness profiles as described in the Appendix A of Pfrommer \& Enßlin (2003). Since Hydra $\mathrm{A}$ is known to exhibit a strong cooling flow as observed in the X-ray studies, we assumed a double $\beta$-profile ${ }^{3}$ and used for the inner profile $n_{\mathrm{e} 1}(0)=0.056 \mathrm{~cm}^{-3}$ and $r_{\mathrm{c} 1}=0.53$ arcmin, for the outer profile we used $n_{\mathrm{e} 2}(0)=0.0063 \mathrm{~cm}^{-3}$ and $r_{\mathrm{c} 1}=2.7$ arcmin and we applied a $\beta=0.77$.

The details of the radio data reduction can be found in Taylor \& Perley (1993). The beamsize of the map is $0.3 \operatorname{arcsec}$ translating into $0.3 \mathrm{kpc}$. The source consists of two lobes, a northern and a southern one. Typical $R M$ values in the north lobe are in the range between $-1000 \mathrm{rad} \mathrm{m}^{-2}$ and $+3300 \mathrm{rad} \mathrm{m}^{-2}$ whereas in the south lobe also values of down to $-12000 \mathrm{rad} \mathrm{m}^{-2}$ were observed.

We concentrated our analysis on the north lobe of this source because the signal-to-noise ratio in the data of the south lobe does not seem sufficient enough for our purposes. Furthermore, the application of the data filter to remove big steps between data pixels leads to a splitting of the $R M$ data in the south lobe into a lot of small spatially disconnected areas. Such a fragmented window function can heavily obscure any power spectrum measured.

For Hydra A, there is a clear depolarisation asymmetry of the two lobes observed as described by the Laing-Garrington effect. Taking this effect into account and following the analysis of Taylor \& Perley (1993), we first rotated the coordinate system about an angle of 110 degrees such that the $x$-axis was parallel to the jet axis. Afterwards the source plane was rotated about the new $y$-axis by an inclination angle $\theta$ of 45 degree such that the north lobe would point towards the observer.

\subsection{Real space analysis}

After the mean $R M$ subtraction and the noisy pixel filtering, the $R M$ autocorrelation function $C_{R M}\left(r_{\perp}\right)$ was determined using Eq. (5) choosing the described normalisation scheme. The resulting correlation function for Abell 2634, Abell 400 and Hydra A are exhibited in Fig. 1, where the correlation function of Hydra A was divided by 100 for better display. The integration of these functions employing Eq. (12) leads to the information on the $R M$ correlation length $\lambda_{R M}$ which was determined to be $7.9 \mathrm{kpc}$ for Abell 2634, $5.3 \mathrm{kpc}$ for Abell 400, and $1.9 \mathrm{kpc}$ for Hydra A.

The determination of the magnetic autocorrelation function in real space $w(r)$ achieved by the deprojection of the $R M$ autocorrelation is numerically difficult due to the term in the denominator $\sqrt{y^{2}-r^{2}}$ of Eq. (9) and Eq. (10) which represents an integrable singularity and can in principle be avoided by a well selected coordinate transformation.

However, the numerical calculation, especially the determination of the value for $r=0$ of the magnetic autocorrelation function $w(r)$ is difficult because an extrapolation to $r=0$ of the function $w(r)$ being itself subject to extrapolation and data

\footnotetext{
${ }^{3}$ Defined as $n_{\mathrm{e}}(r)=\left[n_{\mathrm{e} 1}^{2}(0)\left(1+\left(r / r_{\mathrm{c} 1}\right)^{2}\right)^{-3 \beta}+n_{\mathrm{e} 2}^{2}(0)(1+\right.$ $\left.\left.\left(r / r_{\mathrm{c} 2}\right)^{2}\right)^{-3 \beta}\right]^{1 / 2}$.
}

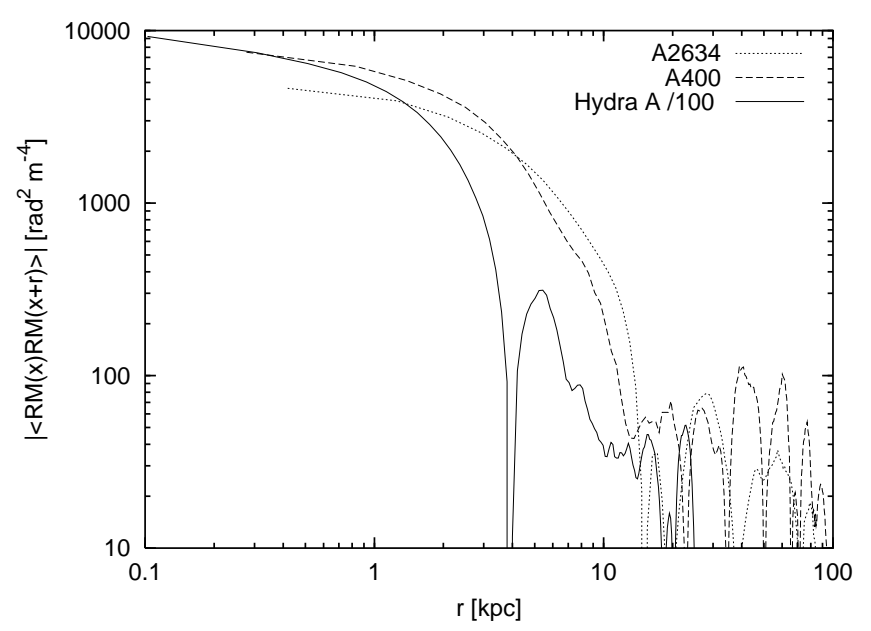

Fig. 1. $R M$ autocorrelation function $\left|C_{R M}\left(r_{\perp}\right)\right|$ of Abell 400, Abell 2634 and Hydra A obtained using Eq. (5). Note that the correlation function for Hydra A was divided by 100 for representing purposes.

binning is involved. Therefore the determination of the point $w(r=0)$ is not accurate and one should be careful with the interpretation of the values derived by this method. The behaviour of the magnetic autocorrelation around $r=0$ is exhibited in Fig. 2 for the case of Abell 2634, where the magnetic field autocorrelation functions $w(r)$ derived by employing various methods are shown. One can see that the steeper the initial slope of the function the less precise it becomes to determine $w(r=0)$ since slight deviations in the slope can lead to very different results.

One should keep this in mind when calculating the value of the magnetic field autocorrelation length $\lambda_{B}$ which was determined to be $6.0 \mathrm{kpc}$ for Abell 2634, $3.9 \mathrm{kpc}$ for Abell 400 and $1.4 \mathrm{kpc}$ for Hydra A using Eq. (14), where for the value of $w(r=0)=B_{0}^{2}$ the extrapolation of Eq. (9) to $r=0$ was used. Thus, the field strengths were determined to be of about $4 \mu \mathrm{G}$ for Abell 2634, of about $9 \mu \mathrm{G}$ for Abell 400 and of about $12 \mu \mathrm{G}$ for Hydra A in the real space analysis.

\subsection{Fourier space analysis}

For the calculations in Fourier space a 2-dimensional Fast Fourier Transform (FFT) algorithm was employed while setting all blanked values in the original $R M$ map to zero. The $R M$ autocorrelation function $\hat{C}_{\perp}\left(k_{\perp}\right)$ was then obtained by summing over rings in $k$-space. Since the magnetic field autocorrelation function $\hat{w}(k)$ is related to the $R M$ autocorrelation function in Fourier space simply by multiplying the $R M$ correlation function $\hat{C}_{\perp}\left(k_{\perp}\right)$ by two (Eq. (16)), there is no numerical difficulty involved deriving this function and thus, the results for the magnetic field quantities are more precise than those obtained applying the real space analysis.

The integration of the power spectrum $\hat{w}^{\text {obs }}(k)$ following Eq. (19) and Eq. (20) yields the correlation lengths. Thus, the $R M$ autocorrelation length $\lambda_{R M}$ was calculated to be $8.0 \mathrm{kpc}$ for Abell 2634, $5.3 \mathrm{kpc}$ for Abell 400 and 2.0 for Hydra A. The magnetic field correlation length $\lambda_{B}$ was determined to be 

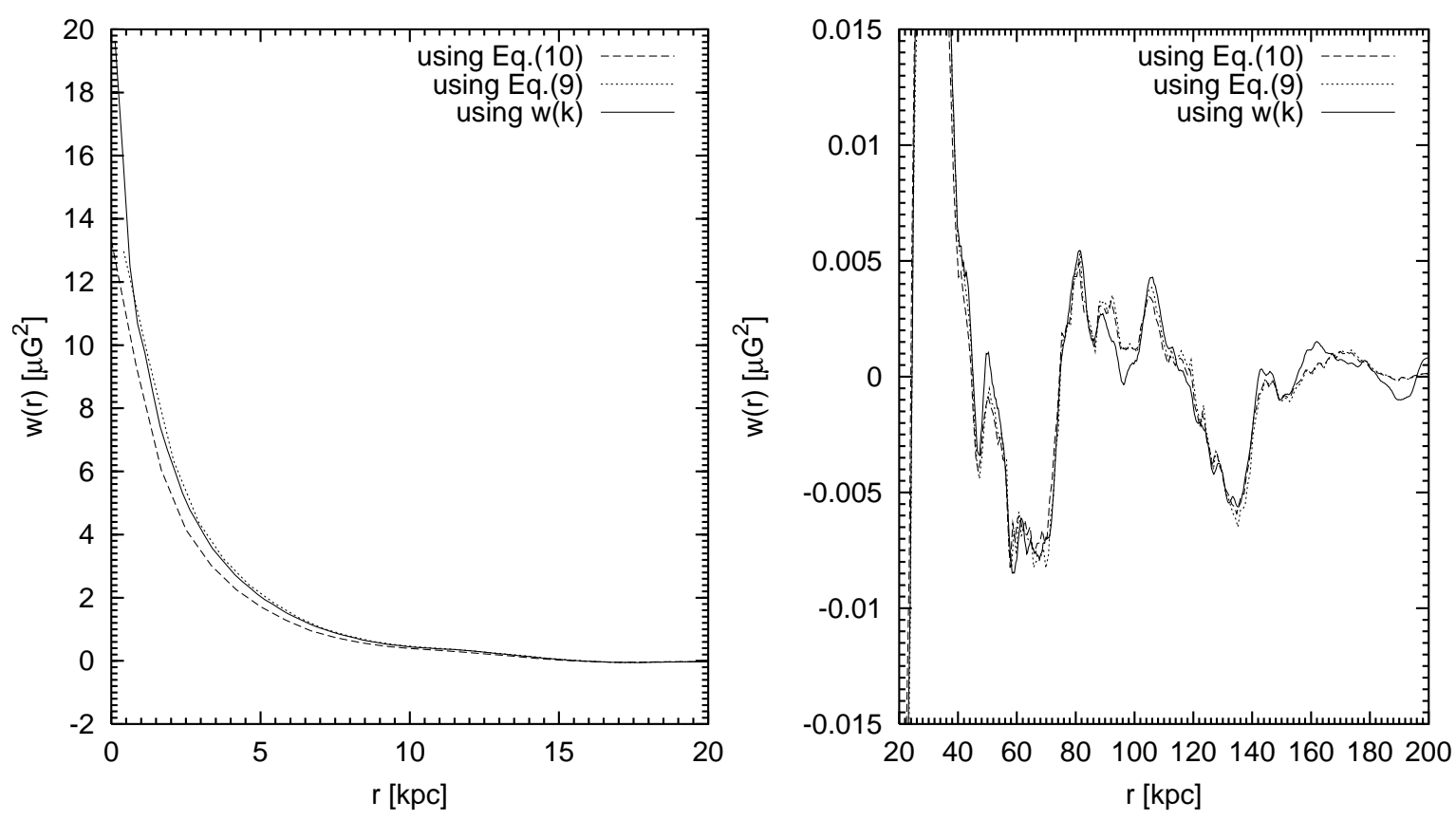

Fig. 2. The magnetic autocorrelation function $w(r)$ for Abell 2634 calculated using Eqs. (9), (10) and (21). One can clearly see that the value of $w(r=0)$ can deviate by a factor of two but for larger $r$ all methods yield almost the same results.

$4.0 \mathrm{kpc}$ for Abell 2634, $2.3 \mathrm{kpc}$ for Abell 400 and $0.5 \mathrm{kpc}$ for Hydra A in the Fourier analysis.

No numerical problem is involved in the determination of the value of the magnetic autocorrelation function $w(r)$ for $r=0$ if one uses Eq. (21) resulting in magnetic field strengths of about $5 \mu \mathrm{G}$ for Abell 2634, of about $11 \mu \mathrm{G}$ for Abell 400 and of about $23 \mu \mathrm{G}$ for Hydra $\mathrm{A}$.

The application of Eq. (21) to the data translates the magnetic autocorrelation function in Fourier space to real space. The comparison of the so derived function with the deprojected functions $w(r)$ in real space is exhibited in Fig. 2 for the case of Abell 2634. One can clearly see that at the origin there are deviations by a factor of two due to the uncertainties connected to the extrapolation used in the real space approach. At higher separations $r$ all three functions shown do not differ significantly from each other. It is remarkable that except at the origin all three independent approaches are in such good agreement which demonstrates reliable numerics.

The knowledge of the 3-dimensional power spectrum $\hat{w}(k)$ also allows calculating the magnetic energy spectrum $\varepsilon_{B}^{\mathrm{obs}}(k)$ by employing Eq. (17). The results for the three clusters are shown in Fig. 3. The magnetic energy spectra are suppressed at small $k$ by the limited window size and the subtraction of the mean $R M$ (small $k$ in Fourier space translate into large $r$ in real space). A response analysis as suggested above is performed in Sect. 3.4 in order to understand this influence in more detail.

Another feature of these energy spectra is the increasing energy density at the largest $k$-vectors in Fourier space and thus, small $r$ in real space. They can be explained by uncorrelated noise on scales smaller than the beamsize which possesses a power spectrum of $\varepsilon_{B} \propto k$ (Enßlin \& Vogt 2003). The determination of the increasing slope of the energy densities for large $k$ revealed proportionalities of $\varepsilon_{B} \propto k^{1.0 \ldots 1.2}$ also shown

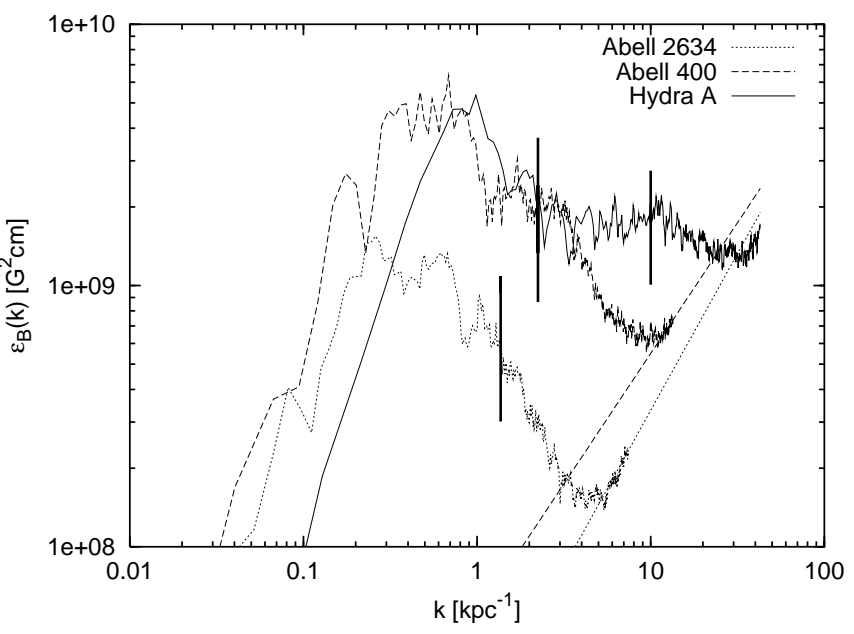

Fig. 3. Magnetic energy spectrum $\varepsilon_{B}^{\text {obs }}(k)$ derived for Abell 400, Abell 2634 and Hydra A. The Fourier transformed beamsizes $\left(k_{\text {beam }}=\right.$ $\left.\pi / l_{\text {beam }}\right)$ are represented by the thick vertical lines. The other straight lines describe the slope (dashed line represents $\varepsilon_{B} \propto k$ and the dotted represents $\varepsilon_{B} \propto k^{1.2}$ ) of the increase in the energy density for the largest $k$.

in Fig. 3. Thus, we conclude that at the largest $k$-scales uncorrelated noise dominates the shape of the energy spectra.

It seems reasonable to introduce an upper cutoff for the integration of the magnetic energy spectra in Eq. (17). In Fig. 3, the equivalent beamsize in Fourier space $k_{\text {beam }}=\pi / l_{\text {beam }}$ (where $l_{\text {beam }}$ is the beamsize in real space defined as $F W H M$ ) is represented by a vertical line for each cluster which is $1.4 \mathrm{kpc}^{-1}$ for Abell 2634, $2.2 \mathrm{kpc}^{-1}$ for Abell 400 and $10.0 \mathrm{kpc}^{-1}$ for Hydra A. One can clearly see that the noise induced increase of the energy density lies on $k$-scales beyond $k_{\text {beam }}$. Therefore, a 


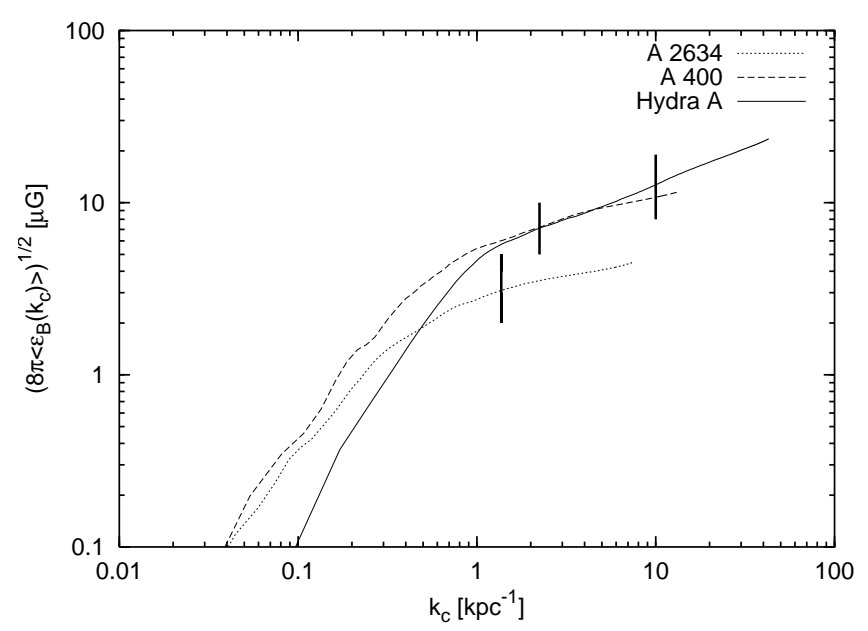

Fig. 4. Magnetic field strength $B$ dependent on the upper $k$-cutoff in the integration of Eq. (17). The equivalent beamsizes $k_{\text {beam }}$ are represented as vertical lines.

suitable upper cutoff for any integration of the magnetic energy spectrum seems to be $k_{\text {beam }}$.

However, the influence of the upper $k$-cutoff in the integration can be seen in Fig. 4 which displays the magnetic field strength $B=\sqrt{8 \pi\left\langle\varepsilon_{B}\right\rangle}$ estimated from

$\varepsilon_{B}\left(k<k_{\mathrm{c}}\right)=\int_{0}^{k_{c}} \mathrm{~d} k \varepsilon_{B}(k)$.

\subsection{Test the model and assess the influence of the window function}

The various possibilities to test the window function and to assess its influences on the results outlined in Sect. 2.1 were also applied to the data. The $\chi^{2}$-function was derived by radially averaging the $\chi^{2}\left(\boldsymbol{x}_{\perp}\right)$ distribution resulting from Eq. (22), where for the magnetic autocorrelation length $\lambda_{B}$ the value derived in the Fourier analysis was used since this approach seems to be more accurate. The resulting radially binned distributions are shown in Fig. 5 determined for the three clusters. There is no apparent spatial large scale trend visible for Abell 400 and Abell 2634 which indicates a reasonable model for the window function. In the case of Hydra A, there appears to be a trend of higher values for $\chi^{2}\left(x_{\perp}\right)$ towards larger $r$, comparable to small scale trends seen in the $\chi^{2}\left(x_{\perp}\right)$ distribution of Abell 2634 and Abell 400.

Furthermore, the $\chi^{2}\left(\boldsymbol{x}_{\perp}\right)$ distribution was integrated following Eq. (23) and $\chi_{a v}^{2}$ was calculated to be 1.0 for Abell 2634, 1.2 for Abell 400 and 1.6 for Hydra A. A refinement of the model describing parameter does not seem to be required for Abell 2634 and Abell 400.

Since the value for $\chi_{a v}^{2}$ of 1.6 for Hydra A is deviating from 1 , we varied the geometry of the source, i.e. we varied the inclination angle $\theta$ between source and observer plane from 30 degree up to 60 degree resulting in field strength ranging from $17 \mu \mathrm{G}$ to $33 \mu \mathrm{G}$ and values for $\chi_{a v}^{2}$ of 1.4 to 1.8 , respectively. These values were derived by integrating over the full accessible unrestricted (without cutoffs) $k$-space.

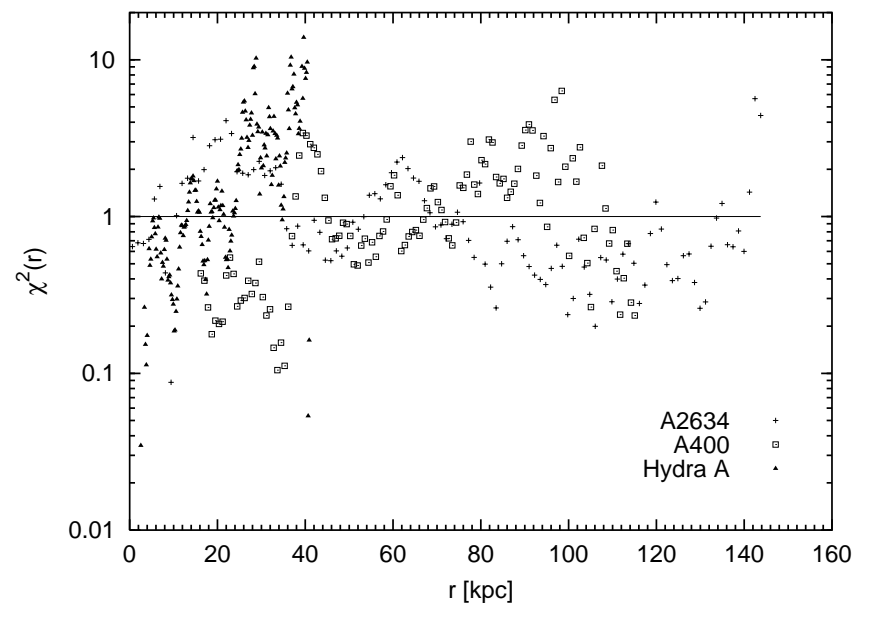

Fig. 5. Testing the window function by calculating the $\chi^{2}\left(x_{\perp}\right)$ distribution for all three cluster.

Furthermore, we varied the scaling parameter $\alpha_{B}$ in the scaling relation of the electron density to the magnetic energy density. A value for $\alpha_{B}$ of 0.5 is still in the limit of reasonable values as suggested by Dolag et al. (2001). Furthermore, such a scaling parameter of $\alpha_{B}=0.5$ means that the magnetic energy $\varepsilon_{B}(x) \propto n_{\mathrm{e}}(\boldsymbol{x})$ and thus, the magnetic field would be proportional to the thermal energy of the cluster gas assuming approximately isothermal conditions. However, in this case $\left(\alpha_{B}=0.5\right)$ one obtains for $\chi_{a v}^{2}$ a value of 1.2 and the magnetic field strength is reduced to $17 \mu \mathrm{G}$ by integrating over the full accessible $k$-space.

One can not be sure that the cause of the trend in the $\chi^{2}\left(x_{\perp}\right)$ distribution is due to a geometry other than assumed because it could also be explained by a fluctuation similar to the one seen in the $\chi^{2}\left(x_{\perp}\right)$ distributions of Abell 2634 and Abell 400. There is no reason to change the initial assumption for the geometry of Hydra A. However, we should keep in mind that the central field strength given could be slightly overestimated for Hydra A but it is not clear to what extent if at all.

A good test for the validity of the isotropy assumption is the inspection of the Fourier transformed $R M$ data $|R \hat{M}(k)|^{2}$ as shown in Fig. 6 for Abell 400 and Hydra A. The FFT data look similar for Abell 2634. In this figure half of the Fourier plane is shown since the other half is symmetric to the one exhibited. No apparent anisotropy is present in this figure.

We mentioned already that the magnetic energy spectrum $\varepsilon_{B}^{\text {obs }}(k)$ in Fig. 3 is suppressed by the limited window size for small $k$-vectors in Fourier space. Therefore a response analysis is necessary in order to understand the influence of the window on the shape of the magnetic energy spectrum. For this purpose, the projected window function was Fourier transformed by employing a FFT algorithm and inserted into Eq. (25). We then compared the response functions $\hat{w}^{\exp }(k) \mathrm{ob}-$ tained to the observed power spectrum $\hat{w}^{\text {obs }}(k)$ as shown in Fig. 7 for the case of Abell 2634, where we chose for the normalisation a magnetic field strength $B$ of $5 \mu \mathrm{G}$. The corresponding figures look very similar for Abell 400 and Hydra A. 

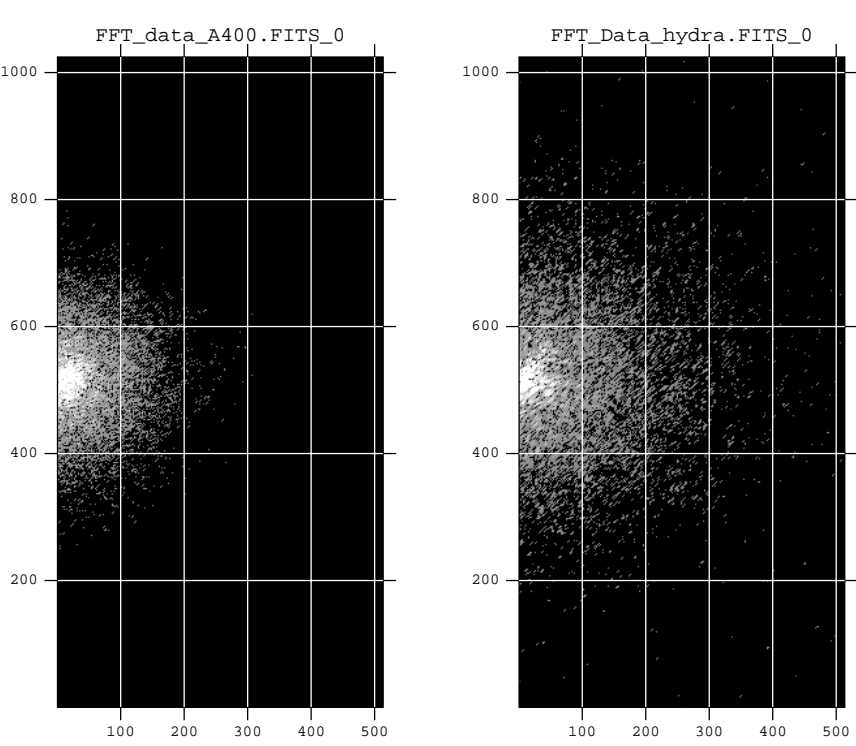

Fig. 6. The power $|\langle R \hat{M}(k)\rangle|^{2}$ of the Fourier transformed $R M$ map of Abell 400 on the left side and Hydra A on the right side. The image for Abell 2634 looks similar. Only half of the Fourier plane is exhibited the other half is point symmetric to the one shown.

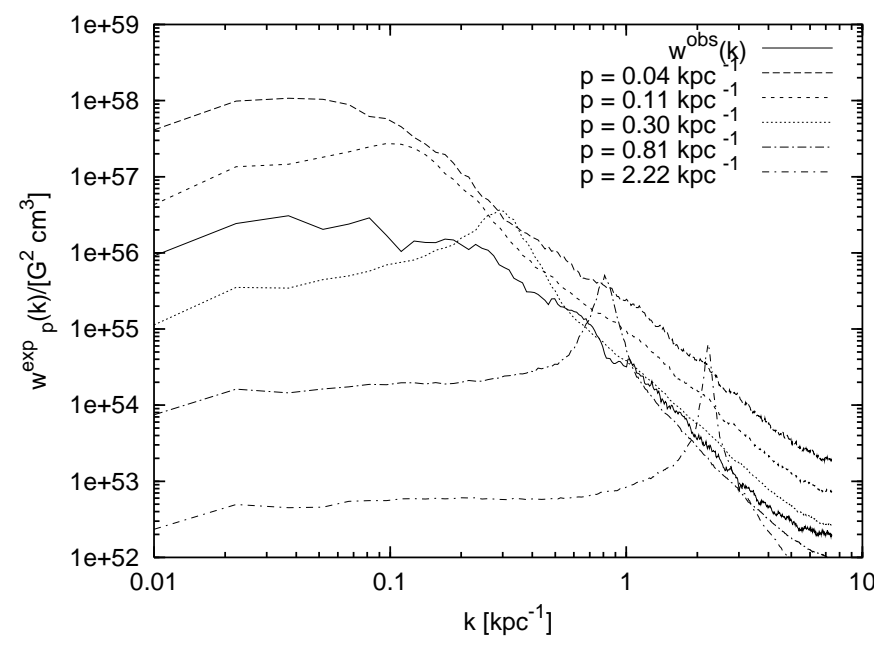

Fig. 7. Responses at various scales $p$ to the window function of Abell 2634 assuming a $B=5 \mu \mathrm{G}$ in comparison to the observed magnetic autocorrelation function $\hat{w}(k)$.

From Fig. 7, one can clearly see that the response to delta function like input power spectra on small $p$ scales in Fourier space (i.e. large $r$ in real space) is a smeared out function as one would expect. The response for larger $p$ becomes more strongly peaked suggesting that at $k$-scales larger than $0.3 \mathrm{kpc}^{-1}$ for Abell 2634 the influence of the window function becomes negligible. From similar plots for the other two clusters under consideration, scales of about $0.4 \mathrm{kpc}^{-1}$ for Abell 400 and $1.0 \mathrm{kpc}^{-1}$ for Hydra A are found. Thus, one would use this value as a lower cutoff $k_{\min }$ for the determination of the magnetic field strength.

From the response power spectra $\hat{w}_{p}\left(k_{\perp}\right)$ calculated for different $p$ requiring a magnetic field strength $B$, one can derive a magnetic field strength $B_{\exp }$ by integration. In Fig. 8, the comparison between these two field strengths is plotted for the

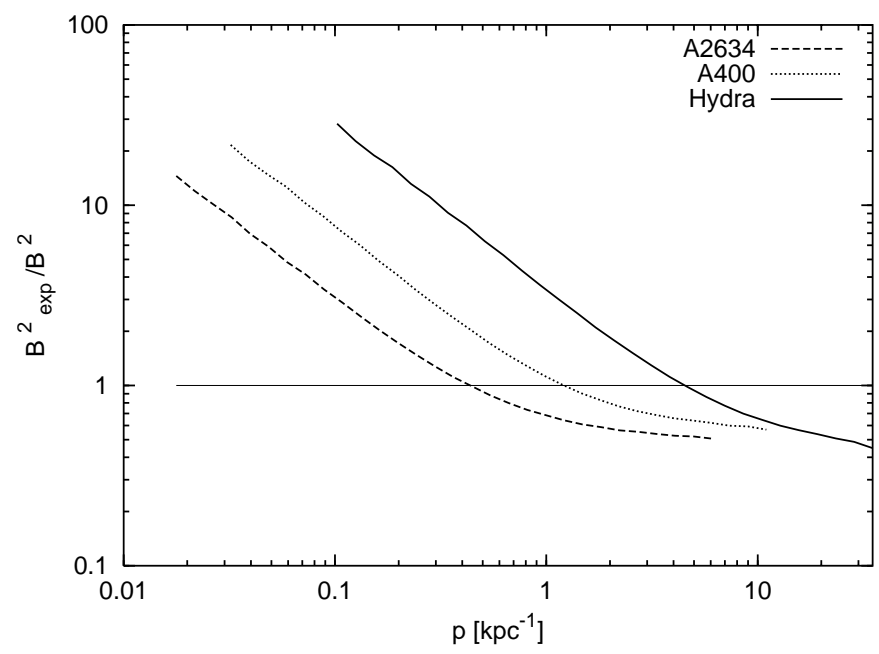

Fig. 8. Comparison between the expected magnetic field strength $B$ to the measured one $B_{\text {exp }}$ for the particular $p$-scales for the three clusters.

different $p$-scales. It can be seen that for the smallest $p$ 's the deviation between expected field strength and actual observed one is significantly but for larger $p$ they are almost equal suggesting that on these scales the influence of the window is negligible. Taking Fig. 8 into account, the values determined above as lower cutoff $k_{\min }$ can be confirmed.

Another possibility to assess the influence of the window on the power spectra is to match the added response functions and the actual observed power spectra $\hat{w}^{\mathrm{obs}}(k)$. This was done by applying Eq. (27) to a set of response functions generated for closely spaced $p$-scales. The resulting function $\hat{w}^{\exp }(k)$ was matched to the actual observed power spectrum $\hat{w}^{\text {obs }}(k)$ for each of the three clusters by varying $B_{0}$, the spectral index $\alpha$ of the power law in Eq. (27) to match the slope of the functions and $p_{\min }$ as lower cutoff to fit the function at its turnover for small $k$-scales. The resulting functions are shown in Fig. 9 in comparison to the respective observed power spectra. The shape of the power spectra was matched for Abell 2634 for a spectral index of $\alpha=1.6$, for Abell 400 for an $\alpha=1.8$ and for Hydra A for an $\alpha=2.0$ whereas a lower $k$-cutoff of $0.09 \mathrm{kpc}^{-1}$ was used for Abell 2634, $0.08 \mathrm{kpc}^{-1}$ for Abell 400 and $0.3 \mathrm{kpc}^{-1}$ for Hydra A.

The spectral index $\alpha$ can also be used to plot the respective power law as represented by the straight lines in Fig. 9 . The slopes of these lines clearly deviate from the respective observed power spectra suggesting that one cannot estimate differential parameters like spectral indeces directly from Fourier transformed $R M$ maps. More sophisticated approaches should be developed. However, our approach allows to exclude flatter spectral slopes than $\alpha=1.3$ still leaving a Kolmogorov spectrum $(\alpha=5 / 3)$ as a possible description. For attempts to measure the magnetic power spectrum from cluster simulations and radio maps see Dolag et al. (2002) and Govoni et al. (2002) respectively ${ }^{4}$.

${ }^{4}$ The conventions describing the spectra may differ in these articles from the one used here. 
Table 1. Values for the autocorrelation length scales $\lambda_{B}$ and $\lambda_{R M}$ and the magnetic field strength $B_{0}$ at the cluster centre obtained in real space analysis, in Fourier space analysis and in restricted Fourier Space analysis are given for the different clusters under consideration. The values are calculated for two different scaling parameters $\alpha_{B}$. Note that for position other than the cluster centre the average magnetic energy is given by $\left\langle\boldsymbol{B}^{2}(\boldsymbol{x})\right\rangle^{1 / 2}=B_{0}\left(n_{\mathrm{e}}(\boldsymbol{x}) / n_{\mathrm{e} 0}\right)^{\alpha_{B}}$.

\begin{tabular}{lccccccccc}
\hline \hline & \multicolumn{3}{c}{ Real space } & \multicolumn{3}{c}{ Non-restricted Fourier space } & \multicolumn{2}{c}{ Restricted Fourier space } \\
Cluster & $\alpha_{B}$ & $\lambda_{R M}[\mathrm{kpc}]$ & $\lambda_{B}[\mathrm{kpc}]$ & $B_{0}[\mu \mathrm{G}]$ & $\lambda_{R M}[\mathrm{kpc}]$ & $\lambda_{B}[\mathrm{kpc}]$ & $B_{0}[\mu \mathrm{G}]$ & $\lambda_{B}[\mathrm{kpc}]$ & $B_{0}[\mu \mathrm{G}]$ \\
\hline Abell 400 & 1.0 & 5.2 & 3.9 & 8 & 5.3 & 2.3 & 12 & 3.6 & 6 \\
Abell 2634 & 1.0 & 7.9 & 6.0 & 4 & 8.0 & 4.0 & 5 & 4.9 & 3 \\
Hydra A & 1.0 & 1.9 & 1.4 & 13 & 2.0 & 0.5 & 23 & 0.9 & 13 \\
\hline Abell 400 & 0.5 & 5.2 & 3.9 & 7 & 5.3 & 2.3 & 10 & 3.4 & 6 \\
Abell 2634 & 0.5 & 7.9 & 6.0 & 3 & 8.0 & 4.0 & 4 & 4.9 & 3 \\
Hydra A & 0.5 & 1.9 & 1.4 & 10 & 2.0 & 0.5 & 17 & 0.9 & 9 \\
\hline
\end{tabular}

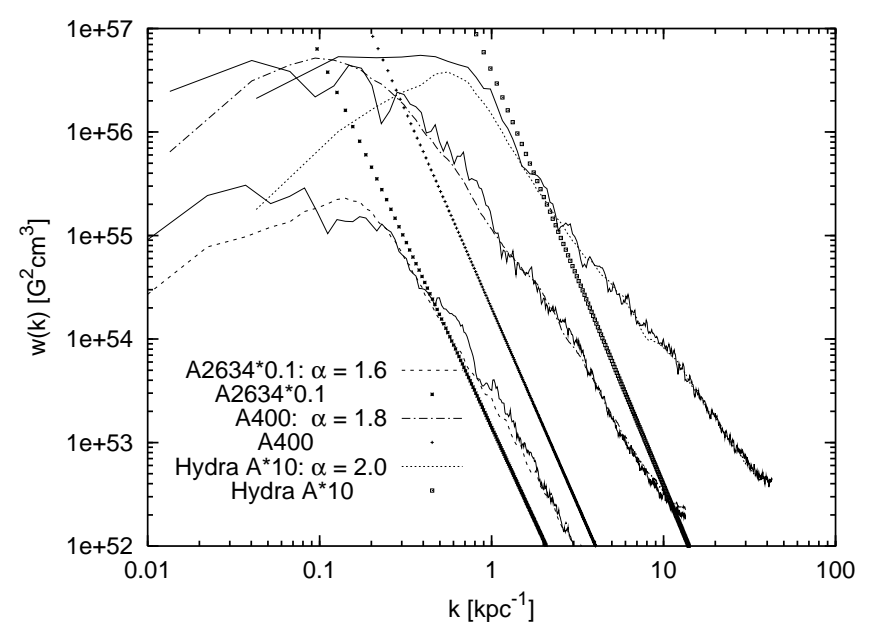

Fig. 9. Closely $p$-spaced response functions were integrated by employing Eq. (27) and the resulting $\hat{w}^{\exp }(k)$ are exhibited in comparison to the observed power spectrum $\hat{w}^{\text {obs }}(k)$ shown as solid lines. The spectral index $\alpha$ used for Abell 2634 is 1.6, for Abell 400 is 1.8 and for Hydra $\mathrm{A}$ is 2.0. The straight lines represent the power law assuming the spectral index determined from the response analysis. Note that the data for Abell 2634 are multiplied by 0.1 and the data for Hydra by 10 for representing purposes.

\section{Results}

A summary of the values for $R M$ autocorrelation length $\lambda_{R M}$, magnetic field autocorrelation length $\lambda_{B}$ and the central magnetic field strength $B_{0}$ for the magnetised cluster gas in Abell 2634, Abell 400 and Hydra A, derived in Fourier space and real space is given in Table 1 . The $R M$ autocorrelation length $\lambda_{R M}$ determined in both spaces is almost equal which demonstrates reliable numerics. Furthermore, it is found that the $R M$ autocorrelation length $\lambda_{R M}$ is larger than the magnetic autocorrelation length $\lambda_{B}$. The deviation of the magnetic field quantities between both spaces is caused by the numerically complicated deprojection of the magnetic autocorrelation function $w(r)$ in real space. Thus, the results in Fourier space are more reliable and should be considered for any interpretation and discussion.

In Sect. 3.4, we discussed many possible influences on the power spectra. We verified that for intermediate $k$-ranges extending over at least one order of magnitude the power spectra are not governed by the window or the resolution of the
$R M$ maps and thus, represent most likely the magnetic field properties of the cluster gas. Thus, one can derive field quantities such as magnetic field strength $B_{0}$ and autocorrelation length $\lambda_{B}$ from this intermediate $k$-range.

The suitable cutoffs for the integration of the power spectra in order to derive the magnetic field strength and autocorrelation length were determined in Sect. 3.4. Using for Abell 2634 a lower cutoff $k_{\min }$ of $0.3 \mathrm{kpc}^{-1}$ and an upper cutoff of $1.4 \mathrm{kpc}^{-1}$ results in a magnetic field strength of $3 \mu \mathrm{G}$. Integrating the power spectrum of Abell 400 in the range from $0.4 \mathrm{kpc}^{-1}$ to $2.2 \mathrm{kpc}^{-1}$ yields a field strength of $6 \mu \mathrm{G}$. The same was done for the Hydra A cluster in the range from $1 \mathrm{kpc}^{-1}$ to $10 \mathrm{kpc}^{-1}$ which resulted in a field strength of $13 \mu \mathrm{G}$. The determination of the magnetic field autocorrelation length $\lambda_{B}$ by integration over the power spectra in limited $k$-space yields $4.9 \mathrm{kpc}$ for Abell 2634, 3.6 kpc for Abell 400 and $0.9 \mathrm{kpc}$ for Hydra A.

In the course of the response analysis in order to estimate the influence of the window on the power spectra, we matched the integrated response power spectra $\hat{w}^{\exp }(k)$ with the actual 3-dimensional observed power spectra $\hat{w}^{\text {obs }}(k)$ and determined the spectral index $\alpha, c_{0}$ and the lower cutoff $p_{\min }$ in Eq. (27). Using these values for the parameters in Eq. (28), the direct integration in the limits of $p_{\text {min }}$ and $p_{\max }=k_{\text {beam }}$ of the power spectra obtained by the response analysis results in magnetic field strength of about $3 \mu \mathrm{G}$ for Abell 2634, of about $6 \mu \mathrm{G}$ for Abell 400 and of about $11 \mu \mathrm{G}$ for Hydra A. These field strengths are in good agreement with the results obtained by the restricted Fourier space integration of the observed 3-dimensional power spectra.

However, the values for $\lambda_{B}$ were determined for the same $p_{\min }$ and $p_{\max }=k_{\text {beam }}$ applying Eq. (29). The resulting value is $13 \mathrm{kpc}$ for Abell 2634, $17 \mathrm{kpc}$ for Abell 400 and $4 \mathrm{kpc}$ for Hydra A. They deviate by a factor of 4 to 5 from the magnetic field autocorrelation length $\lambda_{B}$ determined from the restricted Fourier space integration. The reason for this behaviour could be found in the sensitivity to the lower cutoff $p_{\min }$ and the power law index $\alpha$. We have already mentioned that our method might not be suitable at that stage to determine differential parameters such as a power law index $\alpha$. A more detailed discussion which is beyond the scope of this paper is necessary in order to understand this behaviour.

However, the values of the magnetic field strength $B_{0}$ for Abell 400 and Abell 2634 of about $6 \mu \mathrm{G}$ and $3 \mu \mathrm{G}$, respectively, 
are larger compared to the initial analysis performed by Eilek \& Owen (2002) for the case of a magnetic sheet with a thickness of 10-20 kpc. They discuss a variety of magnetic field models and we refer to Table 2 of their paper for detailed numbers and description.

For the Hydra A cluster, Taylor \& Perley (1993) conclude in their analysis that there is a random magnetic field component of about $30 \mu \mathrm{G}$ with a correlation length of $4 \mathrm{kpc}$. The deviation by a factor of 3 of the field strength from the value revealed by our analysis might be explained by the usage of an improved electron density profile for our analysis which also takes the cooling flow into account. Our value for the central magnetic field strength $B_{0}$ might be also lower due to the conservative approach of restricting the $k$-space integration range. Another explanation for the difference could be sought in our exclusion of the south lobe from the calculation above. Including the south lobe in our analysis leads to higher central field strength but given the influence of the very complicated window function in the case of the south lobe, it is not clear to what extent the real power spectrum is resembled.

The estimation of the dynamical importance of the magnetic fields derived for the cluster gas can be done by comparing the thermal pressure $\left(p_{\text {th }}=2 n_{\mathrm{e}}(0) k T_{\text {core }}\right)$ with the magnetic pressure $\left(p_{B}=B_{0}^{2} /(8 \pi)\right)$. One can calculate $p_{B} / p_{\text {th }}$ which yields 0.08 for the case of Abell 2634 (assuming a $T_{\text {core }}=$ $1.2 \mathrm{keV}$, Schindler \& Prieto 1997), $p_{B} / p_{\text {th }}=0.19$ for Abell 400 $\left(T_{\text {core }}=1.5 \mathrm{keV}\right.$, Eilek \& Owen 2002) and $p_{B} / p_{\text {th }}=0.01$ for Hydra A $\left(T_{\text {core }}=2.7 \mathrm{keV}\right.$, David et al. 2001). It is astonishing that the value of $p_{B} / p_{\text {th }}$ is smaller for Hydra A, which is a cooling flow cluster, than for the non-cooling flow cluster Abell 400 and Abell 2634. The values of $p_{B} / p_{\text {th }}=0.1 \ldots 0.2$ for the latter two clusters give an indication that for those clusters the magnetic field is of some weak dynamical importance for the cluster gas.

\section{Conclusions}

We presented the application of a new analysis of Faraday rotation maps recently developed by Enßlin \& Vogt (2003) to observational data in order to estimate magnetic field strength and autocorrelation length and in order to determine the magnetic power spectra of the magnetised intra-cluster gas. We described that the analysis relies on the assumption that the magnetic fields are statistically isotropically distributed throughout the Faraday screen. We introduced the window function through which any virtually statistically homogeneous magnetic field can be thought to be observed. This window function describes the geometry of the source and the global properties of the intra-cluster gas such as the electron density, known from X-ray measurements, and the global average magnetic field distribution, which we assume to scale with the electron density. Furthermore, we explained two possible approaches in real and Fourier space and outlined the tests for the evaluation of any influence especially arising from the observational nature of the data such as limited source size, resolution and pixel noise on the results obtained. However, we stated that our analysis allows to measure average magnetic energies but it is not sensitive to the particular realisation of the magnetic field structure.
We applied this approach for the first time to observational data and derived not only reliable results for magnetic field strength and autocorrelation length but also we got insight into strength and quality of any results obtained by applying this new analysis to any observational data. In order to understand the possible impacts on the results we reanalysed the Faraday rotation maps of three extragalactic extended radio sources, i.e. 3C 75 in the cluster Abell 400, 3C 465 in Abell 2634, which were kindly provided by Frazer Owen and Jean Eilek, and Hydra A in Abell 780, which was kindly provided by Greg Taylor. We performed the analysis in real and Fourier space for these three Faraday rotation maps. While discussing the difficulties involved in the application to the data, we realised that the calculations in Fourier space are more reliable.

We tested the isotropy assumption and no indication of anisotropy was found. Furthermore a $\chi^{2}$-test was performed in order to assess the model adopted for the geometry of the sampling volume incorporating the global electron density and average magnetic energy distribution. In the case of Abell 2634 and Abell 400, no indication was found that our model may be incorrect. However, in the case of Hydra A we found indications that the window function needs refinement but the indications were not strong enough in order to enforce this refinement.

We realised that the magnetic energy spectra $\varepsilon_{B}(k)$ of the three clusters investigated are dominated on the largest $k$-scales (i.e. smallest $r$-scales) by uncorrelated noise. Therefore it seemed natural to introduce a higher $k$-cutoff $k_{\max }$ for any integration in $k$-space necessary to derive magnetic field quantities such as field strength and autocorrelation length. Being conservative, we used as higher $k$-cutoff the equivalent beamsize in Fourier space $k_{\max }=\pi / l_{\text {beam }}$ which is equal to $1.4 \mathrm{kpc}^{-1}$ for Abell 2634, $2.2 \mathrm{kpc}^{-1}$ for Abell 400 and $10 \mathrm{kpc}^{-1}$ for Hydra A. This will have the effect of loosing some power which is redistributed due to the window from smaller to these larger $k$-scales.

On the smallest $k$-scales, i.e. the largest $r$-scales, power is suppressed because of the limited size of the window. For the assessment of the influence of this suppression on the power spectra, we applied a response analysis described in Sect. 2.4 to the observational windows. The response of the window to delta like input power spectra on small $p$-scales is a wide, smeared out function whereas the response on larger $p$-scales is a peaked function. This observation motivated the introduction of a lower $k$-cutoff $k_{\min }$ in any integration in $k$-space. This value was determined to be $0.3 \mathrm{kpc}^{-1}$ for Abell 2634, $0.4 \mathrm{kpc}^{-1}$ for Abell 400 and $1.0 \mathrm{kpc}^{-1}$ for Hydra A. However, the magnetic field strengths derived are not sensitive to this lower cutoff due to the small $k$ power suppression.

We verified that the intermediate $k$-ranges between $k_{\min }$ and $k_{\max }$ extending over one order of magnitude can be used to determine actual magnetic field properties of the intracluster gas.

Integrating over the response power spectra on particular $p$-scales enables us to match the so determined power spectra and the actually observed power spectra in order to have an independent estimate for magnetic field strengths and spectral slopes. For the three clusters under consideration, spectral 
indices $\alpha$ for the slopes of the power spectra were determined to be in the range from 1.6 to 2.0 . Therefore it would be possible that the magnetic field in these clusters have a Kolmogorov power spectrum exhibiting $\alpha=5 / 3$. However, presently we can not exclude steeper spectra but flatter spectra exhibiting slopes smaller than $\alpha=1.3$ would have been recognised by our analysis. Although we realised that this analysis is not suitable for the determination of differential parameters such as the spectral slopes of power spectra directly from Fourier transformed $R M$ maps, the determination of integrated quantities such as the magnetic field strength appears feasible.

Taking all these arguments into account we determined by integration of the magnetic energy spectrum in the limits between $k_{\min }$ and $k_{\max }$ values for the $R M$ autocorrelation length $\lambda_{R M}$, the magnetic field autocorrelation length $\lambda_{B}$ and the central average magnetic field strength $B_{0}$. An overview of these numbers is given in Table 1 where these values are compared to those derived in real space and non-restricted Fourier space.

The magnetic field autocorrelation length $\lambda_{B}$ was determined for the restricted Fourier space integration to be $4.9 \mathrm{kpc}$ for Abell 2634, $3.6 \mathrm{kpc}$ for Abell 400 and $0.9 \mathrm{kpc}$ for Hydra A. In comparison with the $R M$ autocorrelation length $\lambda_{R M}$ calculated to be $8.0 \mathrm{kpc}$ for Abell 2634, $5.3 \mathrm{kpc}$ for Abell 400 and $2.0 \mathrm{kpc}$ for Hydra A, it can be said that these two characteristic length scales differ from those often assumed in previous work.

The magnetic field strength in the cluster centre $B_{0}$ was calculated for the same limited $k$-space and was determined to be $3 \mu \mathrm{G}$ for Abell 2634, $6 \mu \mathrm{G}$ for Abell 400 and $13 \mu \mathrm{G}$ for Hydra A. Given the assumption of isotropy and a scaling parameter $\alpha_{B}=1.0$, these are conservative values. The resulting magnetic pressures suggest some small but non negligible pressure support for a dynamical influence in the case of Abell 2634 and Abell 400 since the value of $p_{B} / p_{\text {th }}$ is 0.08 and 0.19 , respectively.

We note, that our approach so far is not able to separate the influence of noise on the maps from the astrophysically interesting signal of intra-cluster magnetic fields. Investigations aiming to detect and minimise such spurious signals are in preparation (Enßlin et al. 2003; Dolag et al., in prep.).

Acknowledgements. We thank Jean Eilek, Frazer Owen and Greg Taylor who kindly provided us the Faraday rotation maps of $3 \mathrm{C} 75$, 3C 465 and Hydra A. We acknowledge lively discussions with Greg Taylor, Frazer Owen, Jean Eilek, Tracy Clarke and Christoph Pfrommer. We thank Matthias Bartelmann for comments on the manuscript. C.V. likes to thank for the hospitality at the National Radio Astronomy Observatory (NRAO) in Soccorro in summer 2002, where some of this work was done and lots of interesting discussions took place. C.V. would like to thank the Deutscher Akademischer Austauschdienst (DAAD) for granting a "DAAD Doktorandenstipendium" during the time of the stay at NRAO. This research has made use of the NASA/IPAC Extragalactic Database (NED) which is operated by the Jet Propulsion Laboratory, California Institute of Technology, under contract with the National Aeronautics and Space Administration. This work was done in the framework of the EC Research and Training Network The Physics of the Intergalactic medium.

\section{References}

Bagchi, J., Pislar, V., \& Lima Neto, G. B. 1998, MNRAS, 296, L23

Bicknell, G. V., Cameron, R. A., \& Gingold, R. A. 1990, ApJ, 357, 373

Brunetti, G., Setti, G., Feretti, L., \& Giovannini, G. 2001, MNRAS, 320,365

Carilli, C. L., \& Taylor, G. B. 2002, ARA\&A, 40, 319

Clarke, T. E., Kronberg, P. P., \& Böhringer, H. 2001, ApJ, 547, L111

David, L. P., Nulsen, P. E. J., McNamara, B. R., et al. 2001, ApJ, 557, 546

de Vaucouleurs, G., de Vaucouleurs, A., Corwin, H. G., et al. 1991, Third Reference Catalogue of Bright Galaxies, vol. 1-3, XII (Berlin, Heidelberg, New York: Springer-Verlag)

Dolag, K., Bartelmann, M., \& Lesch, H. 2002, A\&A, 387, 383

Dolag, K., Schindler, S., Govoni, F., \& Feretti, L. 2001, A\&A, 378, 777

Dreher, J. W., Carilli, C. L., \& Perley, R. A. 1987, ApJ, 316, 611

Eilek, J. A., \& Owen, F. N. 2002, ApJ, 567, 202

Enßlin, T. A., \& Biermann, P. L. 1998, A\&A, 330, 90

Enßlin, T. A., \& Vogt, C. 2003, A\&A, 401, 835

Enßlin, T. A., Vogt, C., Clarke, T. E., \& Taylor, G. B. 2003, ApJ, in press [astro-ph/0301552]

Felten, J. E. 1996, in Clusters, Lensing, and the Future of the Universe, ASP Conf. Ser., 88, 271

Feretti, L. 1999, in Diffuse Thermal and Relativistic Plasma in Galaxy Clusters, 3

Feretti, L., Dallacasa, D., Giovannini, G., \& Tagliani, A. 1995, A\&A, 302, 680

Feretti, L., Dallacasa, D., Govoni, F., et al. 1999, A\&A, 344, 472

Fusco-Femiano, R., dal Fiume, D., Feretti, L., et al. 1998, in Abstracts of the 19th Texas Symp. on Relativistic Astrophysics and Cosmology, Paris, France, Dec. 14-18, 1998, ed. J. Paul, T. Montmerle, \& E. Aubourg (CEA Saclay)

Garrington, S. T., \& Conway, R. G. 1991, MNRAS, 250, 198

Garrington, S. T., Leahy, J. P., Conway, R. G., \& Laing, R. A. 1988, Nature, 331, 147

Ge, J. P., \& Owen, F. N. 1993, AJ, 105, 778

Govoni, F., Feretti, L., Murgia, M., et al. 2002, in ASP Conf. Ser., Matter and Energy in Clusters of Galaxies [astro-ph/0211292]

Govoni, F., Taylor, G. B., Dallacasa, D., Feretti, L., \& Giovannini, G. 2001, A\&A, 379, 807

Harris, D. E., \& Grindlay, J. E. 1979, MNRAS, 188, 25

Ikebe, Y., Makishima, K., Ezawa, H., et al. 1997, ApJ, 481, 660

Laing, R. A. 1988, Nature, 331, 149

Markevitch, M., Ponman, T. J., Nulsen, P. E. J., et al. 2000, ApJ, 541, 542

Mohr, J. J., Mathiesen, B., \& Evrard, A. E. 1999, ApJ, 517, 627

Peres, C. B., Fabian, A. C., Edge, A. C., et al. 1998, MNRAS, 298, 416

Perley, R. A., \& Taylor, G. B. 1991, AJ, 101, 1623

Pfrommer, C., \& Enßlin, T. A. 2003, A\&A, in press [astro-ph/0306257]

Reiprich, T. H., \& Böhringer, H. 2002, ApJ, 567, 716

Rephaeli, Y., Gruber, D. E., \& Rothschild, R. E. 1987, ApJ, 320, 139

Rudnick, L., \& Blundell, K. M. 2003, ApJ, 588, 143

Schindler, S., \& Prieto, M. A. 1997, A\&A, 327, 37

Subramanian, K. 1999, Phys. Rev. Lett., 83, 2957

Taylor, G. B., Govoni, F., Allen, S. W., \& Fabian, A. C. 2001, MNRAS, 326, 2

Taylor, G. B., \& Perley, R. A. 1993, ApJ, 416, 554

Vikhlinin, A., Markevitch, M., \& Murray, S. S. 2001, ApJ, 549, L47

Wegner, G., Colless, M., Saglia, R. P., et al. 1999, MNRAS, 305, 259

White, D. A. 2000, MNRAS, 312, 663 